\title{
COMPARACIÓN IN VITRO DE LA RESISTENCIA A LA FRACTURA ANTE CARGA COMPRESIVA Y LA DUREZA DE DOS CERÓMEROS Y DOS RESINAS EMPACABLES CON Y SIN TRATAMIENTO TÉRMICO
}

\author{
${ }^{1}$ Antonio Bonilla Nova, ${ }^{1}$ Yamile Edilma Franco Rueda, ${ }^{1}$ Sandra Milena Lara Puerto, ${ }^{1}$ Daniel Armando Peña Acevedo, \\ ${ }^{2}$ Lucy Margarita Reyes Sarmiento, ${ }^{3}$ Jhon Jairo Gil Pelaez, ${ }^{4}$ Jorge Guillermo Díaz Rodríguez. \\ ${ }^{1}$ Estudiante III año Especialización en Rehabilitación Oral U. Santo Tomás, Bucaramanga (Colombia). \\ ${ }^{2}$ Odontóloga U. Santo Tomás, Especialista en Rehabilitación Oral U. Nacional de Colombia, Docente U. Santo Tomás, Bucaramanga (Colombia) \\ ${ }^{3}$ Ingeniero Mecánico U. del Valle, MSc Mechanical Engineering, PhD Civil Engineering, Docente U. Santo Tomás, Bucaramanga (Colombia). \\ ${ }^{4}$ Ingeniero Mecánico U. Industrial de Santander, Master of Science Mechanical Engineering U. North Texas, Docente U. Santo Tomás, Bucaramanga (Colombia).
}

\begin{abstract}
Auror responsable de correspondencia: Jorge Guillermo Díaz R.
\end{abstract}
Correo electrónico: jorgeguillermo12@mail.ustabuca.edu.co

\section{RESUMEN}

Objetivo: Comparar la resistencia a la fractura ante carga compresiva y dureza de dos cerómeros y dos resinas empacables con y sin tratamiento térmico.

Materiales y métodos: Se realizó un estudio experimental In vitro, cuya muestra estuvo constituida por 120 especímenes distribuidos igualmente en seis grupos: cerómero Adoro-Ivoclar, Gradia-G.C, resina empacable P60-3M fotopolimerizados, fotopolimerizados y sometidos a tratamiento térmico, SureFil-Dentsply fotopolimerizados, fotopolimerizados y sometidos a tratamiento térmico. Se realizó una prueba de compresión en una máquina universal de ensayos con celda de carga de $10 \mathrm{kN}$ a una velocidad $0.5 \mathrm{~mm} / \mathrm{min}$. Para la prueba de dureza Vickers se usó un microdurómetro con carga de $50 \mathrm{gf}$ por 15 segundos. Se realizó análisis univariado y se presentaron los promedios y las desviaciones estándar. Se realizó un análisis bivariado con la prueba ANOVA para cruzar variables.

Resultados: La resina P60 con tratamiento térmico presentó mayor resistencia a la fractura (273,26Mpa) y Adoro (164,13Mpa) mostró el valor más bajo. La resina P60 sin tratamiento térmico presentó mayor dureza (93,62 VHN), el material que presentó menor dureza fue Gradia (35,35 VHN).

Conclusiones: La resina P-60 tiene mayor dureza y mayor resistencia a la fractura. Así mismo, esta resina curada con luz más tratamiento térmico es más resistente a la fractura que cuando es curada únicamente con luz. [Bonilla A, Franco YE, Lara SM, Peña DA, Reyes LM, Gil JJ, Díaz JG. Comparación In vitro de la resistencia a la fractura ante carga compresiva y la dureza de dos cerómeros y dos resinas empacables con y sin tratamiento térmico. Ustasalud 2011; 10: 36 - 42]

Palabras clave: Resina compuesta, Resistencia a la fractura, Pruebas de dureza.

\section{IN VITRO COMPARISON OF RESISTANCE TO FRACTURE COMPRESSIVE LOAD AND STRENGTH OF TWO RESIN-TWO CEROMER PACKED WITH AND WITHOUT HEAT TREATMENT}

\section{ABSTRACT}

Objective: To compare fracture resistance to compressive load and hardness of two ceromers and two packable composite resins with and without heat treatment.

Methods: One hundred and twenty specimens, distributed equally in six groups: ceromer Adoro-Ivoclar, Gradia-G.C, packable composite resin P60- 3M light-curing, light-curing and under heat treatment, SureFil-Dentsply light-curing, light-curing and under heat treatment were evaluated. Compression tests were conducted in a universal test machine with load cell of $10 \mathrm{kN}$ at $0,5 \mathrm{~mm} / \mathrm{min}$. Vickers hardness test were performed in a microdurometer with load of 50 gf during $15 \mathrm{seg}$. Univaried analysis was made to present average and standard deviation, and analysis bivaried with test ANOVA to cross variables.

Results: P60 with heat treatment offered the greatest resistance to the fracture (273.26 MPa) and Adoro (164.13 MPa) showed the lowest value. $\mathrm{P} 60$ without heat treatment presented the highest hardness ( $93.62 \mathrm{VHN})$ whereas the material that presented less hardness was Gradia (35.35 VHN).

Conclusion: P-60 resin has the greatest hardness and resistance to the fracture. The P-60 resin cured with light plus heat treatment is more resistant to the fracture than without heat treatment.

Key words: Composite resins, Resistance to fracture, Hardness tests. 


\section{INTRODUCCIÓN}

La odontología restauradora ha avanzado en los últimos años por las exigencias estéticas y funcionales, se han logrado cambios sustanciales en los materiales dentales y en sus técnicas de aplicación. ${ }^{1}$ Las restauraciones metálicas, por método directo e indirecto fueron la opción de tratamiento por mucho tiempo; sin embargo, las exigencias estéticas han hecho que los materiales dentales deban evolucionar para satisfacerlas.

Las resinas de fotocurado son consideradas una alternativa a la amalgama para restaurar dientes posteriores de forma directa. Según los reportes y documentos de estudios clínicos como el de Sheibenbogen y colaboradores (1999), el 90\% de las restauraciones en posteriores por método directo en resina, fueron clínicamente aceptables en una evaluación a dos años. ${ }^{2}$ Barnes y colaboradores (1991) reportaron $90 \%$ de éxito a cinco años aunque este disminuyó a $77 \%$ a los ocho años. ${ }^{3}$

El uso de las resinas por técnica directa en el sector posterior es limitado debido a sus insuficientes propiedades mecánicas, presentan fallas por contracción de polimerización y baja resistencia al desgaste, que lleva a la pérdida de la forma anatómica bajo abrasión en el proceso masticatorio y a la fractura de los márgenes de la restauración. ${ }^{4}$ Por esto se desarrolló la técnica indirecta en resinas compuestas, para soportar cargas masticatorias con fuerzas de tipo compresivo, tensional y tangencial, mejorar la dureza, la adaptación y el sellado a nivel gingival, controlar la polimerización, evitar el desgaste oclusal y funcional, proveer un contacto proximal duradero y lograr un mejor pulimento. Sin embargo, los resultados de las resinas por método indirecto no fueron óptimos y se desarrollaron los cerómeros, que son materiales restaurativos con propiedades mecánicas superiores a las resinas convencionales, debido a un mayor grado de conversión obtenido por el uso de diferentes procesos de polimerización tales como fotoactivación, calor y/o atmósfera de nitrógeno. ${ }^{5,6}$

La odontología estética ha introducido sistemas restauradores como las resinas compuestas empacables y los cerómeros. Las resinas empacables surgieron por el desarrollo progresivo de los materiales estéticos restaurativos para dientes posteriores. Estas tienen diferentes características, principalmente en la distribución y tamaño de partículas inorgánicas, lo que causa diferencias profundas en las propiedades mecánicas y físicas de estos composites. ${ }^{7}$

Los cerómeros son polímeros optimizados con partículas o cargas cerámicas, por esta razón son catalogados como la séptima generación de resinas compuestas. Son utilizados para diferentes restauraciones por técnica indirecta. ${ }^{8,9}$

Tanto las resinas empacables como los cerómeros son materiales que se caracterizan por tener una matriz inorgánica compuesta de vidrios como zirconio, sílice, bario, flúor, boro silicato, entre otros, de diferentes tamaños que oscilan entre 0.5 y $30 \mu \mathrm{m}$. Además utilizan copolímeros que son composites de micro-relleno fragmentados y molidos, incorporados a las resinas compuestas, como refuerzo de la matriz inorgánica. ${ }^{8,10}$

SureFil y P60 son resinas híbridas, debido a que en su composición de relleno inorgánico contienen partículas de tamaño que oscilan entre 0,6 a 1,0um inmersas en la matriz orgánica, lo que las hace que su indicación sea para restaurar el sector posterior ya que tienen mejores propiedades comparándolas con las primeras resinas convencionales (macro-relleno). ${ }^{7}$

El objetivo de esta investigación fue comparar la resistencia a la fractura y la dureza de dos cerómeros (Gradia-GC y Adoro-Ivoclar) y dos resinas empacables fotopolimerizadas con y sin tratamiento térmico (SureFil-Dentsply, P60-3M).

\section{MATERIALES Y MÉTODOS}

Se realizó un estudio experimental In vitro. La población de este estudio correspondió a especímenes elaborados según la norma ISO 604 (1996) con cada uno de los materiales: dos cerómeros Gradia-GC y Adoro-Ivoclar y dos resinas empacables P60-3M, SureFil-Denstply, con y sin tratamiento térmico.

Se tomaron, según reportes en literatura, 120 especímenes, distribuidos en seis grupos con 20 especímenes cada uno así: cerómero Adoro, cerómero Gradia, resina empacable P60 fotopolimerizados, P60 fotopolimerizados y sometidos a tratamiento térmico (TT), resina empacable SureFil fotopolimerizados y resina empacable SureFil fotopolimerizados y sometidos a TT. Se incluyeron los especímenes de resina empacable y cerómero que cumplieran con las siguientes medidas para la prueba de compresión: $8 \mathrm{~mm}$ de altura y $4 \mathrm{~mm}$ de diámetro y de $2 \mathrm{~mm}$ de altura y $7 \mathrm{~mm}$ de diámetro para la prueba de dureza según la norma ISO 604 (2006).

Se excluyó a los especímenes que no polimerizaron completamente y que presentaron superficies irregulares o porosas. La variable independiente fue el tipo de material: Cerómero y Resina empacable y las variables independientes fue la resistencia a la fractura y dureza. Se fabricaron moldes de teflón con medidas específicas para cada prueba.

La superficie interna de cada molde fue aislada con una película fina de petrolato. El molde fue colocado 
verticalmente en una lámina portaobjetos de microscopio y el material fue condensado por capas de $2 \mathrm{~mm}$ y fotopolimerizadas según las recomendaciones de la casa fabricante. La parte superior del molde fue cubierta por una tira de mylar sobre la cual una lámina portaobjetos fue colocada con presión leve para permitir una superficie lisa. Los especímenes de cerómero (Gradia y Adoro) después de la fotopolimerización inicial por 10 segundos (lámpara GC Steplight SL- Gradia y Luz Quick- Adoro) fueron sometidos a curado final con su respectivo horno (Labolight LV III-Gradia por 5 minutos y Lumamat 100-Adoro por 25 minutos). Los especímenes de resina compuesta empacable (P60- 3M y SureFil- Dentsply) fueron fotopolimerizados con lámpara LED TPC55 por 20 segundos; y la mitad de las muestras fueron adicionalmente sometidas a tratamiento térmico en agua a ebullición $\left(100^{\circ} \mathrm{C}\right)$ por 7 minutos. ${ }^{6}$ Se almacenaron en agua destilada a temperatura ambiente por 8 días. ${ }^{11-13}$

La prueba de compresión fue llevada a cabo en una máquina de ensayos universal (Shidmazu Autograph AG-I $250 \mathrm{kN}$ ) con una celda de carga de $10 \mathrm{kN}$ con una velocidad constante de $0.5 \mathrm{~mm} / \mathrm{min}$ en el laboratorio de resistencia de materiales de la facultad de Ingeniería Mecatrónica de la Universidad Santo Tomás. La adquisición de datos fue realizado por el software Trapezium 2.23 con toma de datos cada 50mseg. La Figura 1 muestra una probeta montada en la máquina universal de ensayos.

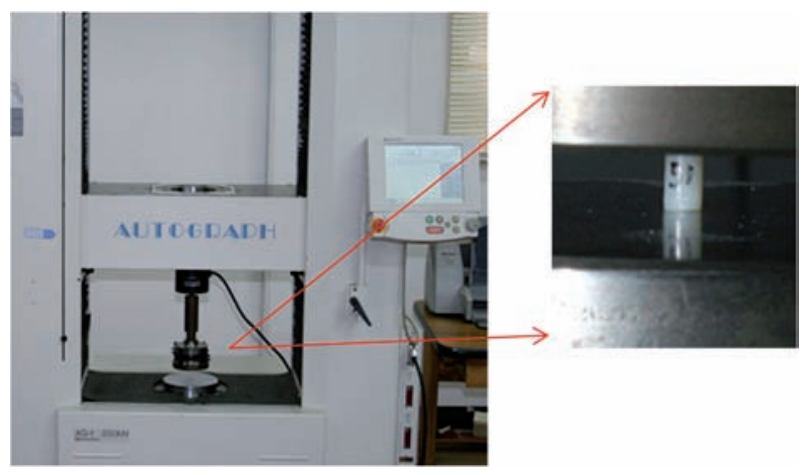

Figura 1. Muestra en ensayo de compresión.

La prueba de dureza Vickers fue conducida mediante un microdurómetro Shimadzu referencia $N^{\circ} 4989$ en el laboratorio de control de calidad del Sena. Las indentaciones fueron hechas bajo una carga de $50 \mathrm{gf}$ por 30 segundos. ${ }^{14,15}$ Se realizaron tres indentaciones en la superficie de cada espécimen. Las dimensiones $l_{1}$ y $l_{2}$ de las indentaciones fueron medidas bajo microscopio y los valores de dureza se determinaron con la fórmula mostrada en la Ecuación 1.

\section{Ecuación 1}

$$
V H N=\left(\frac{1854,4 F}{\left(\frac{l_{1}+l_{2}}{2}\right)^{2}}\right)
$$
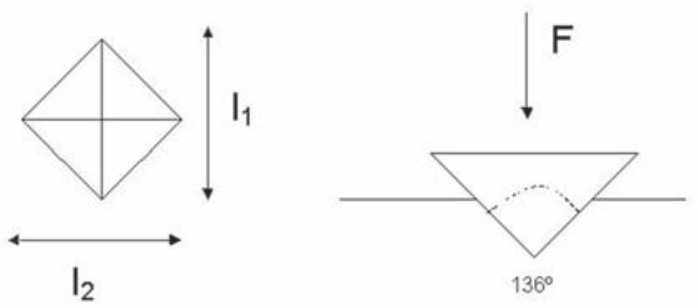

En donde F: Carga aplicada en gramos, $1_{1}$ y $l_{2}$ son las diagonales de la indentación en el material.

Es importante mencionar que algunas muestras mostraron una recuperación falsa. Este fenómeno se presenta en pruebas a compresión cuando una probeta, después de perder su geometría original por fractura, aún soporta cada vez más carga. Para este estudio, y a pesar que dichas probetas aún mostraban una resistencia a la carga, se determinó como punto de fractura la carga que ocasionó fractura en la muestra. Esto debido a consideraciones estéticas y clínicas mas no exclusivamente estructurales. La Figura 2 muestra una curva fuerza vs. desplazamiento en la que el software detectó punto de ruptura en $2,9 \mathrm{kN}$ mas no en $2,3 \mathrm{kN}$.

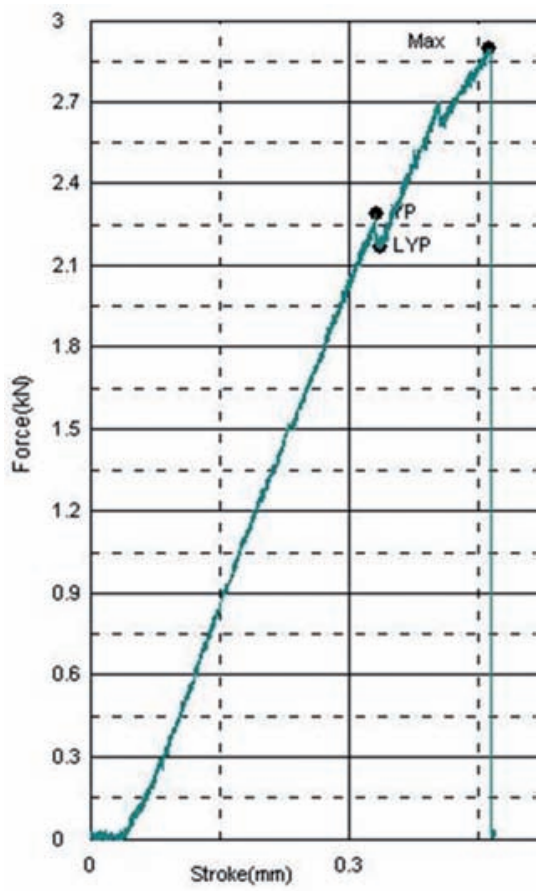

Figura 2. Probeta que muestra recuperación falsa.

Para la recolección de la información se utilizó un formato en donde se registró el tipo de material uti- 
lizado, los datos de resistencia a la fractura y dureza, y el tipo de polimerización a la que fueron sometidos los especímenes.

En el análisis univariado las variables se presentaron como promedios y desviación estándar y en el bivariado se realizó la prueba de Análisis de Varianza, ANOVA, y la prueba de Bonferroni para determinar si había diferencias estadísticamente significativas en la resistencia a la fractura y la dureza, según el tipo material. El presente estudio no representa ningún tipo de riesgo, según la resolución No. 08430 de 1993 del Ministerio de Salud de Colombia en la cual se describe los procedimientos realizados en investigaciones en salud y se clasifican según el nivel de riesgo.

\section{RESULTADOS}

Según los datos experimentales recogidos durante la investigación se determinaron los promedios de los seis grupos de materiales objeto de estudio (Adoro, Gradia, P60 sin tratamiento térmico, P60 con tratamiento térmico, SureFil sin tratamiento térmico, SureFil con tratamiento térmico).

Se presenta el análisis univariado con los promedios y la desviación estándar de la resistencia a la fractura en la Tabla 1.

Tabla 1. Comparación de los valores de resistencia a la fractura a 23C según el tipo de material.

\begin{tabular}{ccccc}
\hline Muestra \# & MATERIAL & Esfuerzo último (MPa) & Desviación estándar & P \\
\hline $1-10$ & Adoro & 164,13 & 38,4 & \\
$11-20$ & P60 TT & 273,26 & 35,79 & \\
$21-30$ & P60 & 254,71 & 51,63 & 0,0000 \\
$31-40$ & Gradia & 172,87 & 35,60 & \\
$41-50$ & SureFil TT & 268,96 & 21,02 & \\
$51-60$ & SureFil & 242,75 & 43,30 & \\
\hline
\end{tabular}

El material que presentó mayor resistencia a la fractura ante carga compresiva fue la resina P60 con tratamiento térmico, con una media $273,26 \mathrm{MPa}$, seguida de la resina SureFil con tratamiento térmico con un promedio de $268,96 \mathrm{MPa}$. El menor promedio de resistencia a la fractura fueron los cerómeros Gradia con 172,87MPa y Adoro con 164,13MPa.
Al realizar la prueba ANOVA, para la resistencia a la fractura según el tipo de material, y que se muestra en la Tabla 2, se encontraron diferencias estadísticamente significativas $(p=0.0000)$. La prueba de ANOVA para la dureza, según el tipo de material, mostró diferencias estadísticamente significativas $(p=0.0000)$.

Tabla 2. Prueba ANOVA para resistencia a compresión.

\begin{tabular}{cccccc}
\hline \multirow{2}{*}{ Fuente de variación } & \multicolumn{5}{c}{ ANOVA } \\
\cline { 2 - 6 } & SS & FD & SM & F & Prob $>$ F \\
Entre grupos & 117629.067 & 5 & 23525.8133 & & \\
Dentro del grupo & 96199.7633 & 54 & 1781.4771 & 13.21 & 0.0000 \\
\hline
\end{tabular}

SS: suma de cuadrados; FD: grados de libertad; SM: Cuadrado de las medias; F: test F.

Al comparar cada tipo de material, mediante la prueba de Bonferroni se encontraron diferencias estadísticamente significativas $(\mathrm{p}=0.000)$ para la prueba de compresión así:

- Adoro y P60, Adoro y P60 con TT, Adoro y Surefil con TT, Gradia y P60 con TT, Gradia y Surefil con TT, Gradia y P60 $(p=0.001)$.

- Adoro y Surefil $(p=0.002)$.

- Gradia y Surefil $(\mathrm{p}=0.008)$.

Los resultados de la prueba de dureza para los seis grupos de materiales se presentan en la Tabla 3. El material que presentó mayor promedio de dureza fue la resina $\mathrm{P} 60$ sin tratamiento térmico con un promedio de 93,628 , seguida por la resina P60 con tratamiento térmico con un promedio de 93,136 , mientras los materiales que presentaron menor dureza fueron los cerómeros Adoro con 51,001 y Gradia con 35,352 de promedio.

La prueba de ANOVA para la dureza, según el tipo de material, mostró diferencias estadísticamente significativas. $(\mathrm{p}=0.0000)$ como lo muestra la Tabla 4. 
Tabla 3. Comparación de los valores de dureza Vickers, según el tipo de material.

\begin{tabular}{cccc}
\hline MATERIAL & MEDIA (VHN) & DESVIACIÓN ESTÁNDAR & p \\
\hline Adoro & 51,00 & 2,86 & \\
Gradia & 35,35 & 2,08 & \\
P60 sin TT & 93,62 & 2,83 & 0,0000 \\
P60 con TT & 93,13 & 2,11 & \\
SureFil sin TT & 69,78 & 2,77 & \\
SureFil con TT & 85,97 & 5,37 & \\
\hline
\end{tabular}

Tabla 4. Prueba ANOVA para dureza Vickers.

\begin{tabular}{cccccc}
\hline \multirow{2}{*}{ Fuente de variación } & \multicolumn{7}{c}{ ANOVA } \\
\cline { 2 - 6 } & SS & FD & SM & F & Prob $>$ F \\
Entre grupos & 28970.4281 & 5 & 5794.08561 & 563.91 & 0.0000 \\
Dentro del grupo & 554.840089 & 54 & 10.2748165 & \\
\hline
\end{tabular}

SS: suma de cuadrados; FD: grados de libertad; SM: Cuadrado de las medias; F: test F.

La comparación de la dureza entre los diferentes tipos de material mostró diferencias estadísticamente significativas $(P=0.000)$ entre: Adoro y Gradia, Adoro y P60, Adoro y P60 con TT, Adoro y Surefil, Adoro y Surefil con TT, Gradia y P60, Gradia y P60 con TT, Gradia y Surefil, Gradia y Surefil con TT, P60 sin tratamiento térmico y Surefil, P60 sin tto. térmico y Surefil con TT, P60 con tto. térmico y Surefil, P60 con tto. térmico y Surefil con TT, Surefil sin tto. térmico y Surefil con TT. No se hallaron diferencias estadísticamente significativas entre P60 y P60 con TT $(\mathrm{P}=1.000)$.

\section{DISCUSIÓN}

En este estudio se evaluaron algunas propiedades mecánicas, como la dureza y la resistencia a la fractura de resinas empacables y cerómeros, manejados como lo describe la casa fabricante para ambos materiales y con polimerización adicional a un grupo de resinas empacables. ${ }^{6,9}$

Los resultados encontrados confirman que el tamaño de las partículas de relleno, su forma y contenido en volumen le confieren al material mejores propiedades físico mecánicas como la dureza y la resistencia a la fractura. Condon y Ferracane en 1997, describen resultados similares. ${ }^{16}$

Sabbagh y colaboradores (2004) describen que las partículas de forma esférica que se observan en la resina P60, mejoran el empaquetamiento de las mismas y permiten aumentar la fracción del volumen del relleno en la resina. Su forma redondeada y homogénea aumenta la resistencia a la fractura del material. Sin embargo, como no todas las partículas de las resinas compuestas y los cerómeros son redondas como en la P60, sino de forma irregular, el stress mecánico tiende a concentrarse en las irregularidades de la interfase de relleno y matriz, ángulos y protuberancias del relleno, que son zonas donde se inician prontamente las fracturas. ${ }^{11}$

Esto soporta los resultados del estudio donde la resina $\mathrm{P} 60$ demostró mayores valores de resistencia a la fractura con un promedio de 273.26MPa comparado con los cerómeros Gradia y Adoro con promedios de 172.87MPa y 164.13Mpa, respectivamente.

Así mismo, los valores más altos de dureza reportados en el estudio corresponden a la resina P-60 (3M) con un valor de 93.62 VHN comparados con los resultados obtenidos por los cerómeros Adoro (51 VHN) y Gradia (35.35 VHN), estos resultados coinciden con el estudio de Da Fonte Porto (2004) quien concluye que la resina Z100, resina híbrida al igual que la P-60, presentó mayor valor de dureza (110 VHN) y resistencia compresiva (275 $\mathrm{MPa}$ ) comparado con cerómeros como el Targis (32 VHN,198 MPa ) y Artglass (50 VHN, $210 \mathrm{MPa}){ }^{11}$

El valor de dureza reportado por la casa fabricante para la resina $\mathrm{P} 60-3 \mathrm{M}$ es de $97,20 \pm 0,26,{ }^{17}$ estos valores coinciden con los obtenidos en esta investigación. Teóricamente, los cerómeros son materiales que presentan un mejor comportamiento mecánico comparado con las resinas. ${ }^{9}$ Sin embargo, los resultados de este estudio no coincidieron con esta premisa, puesto que el comportamiento de los materiales depende de la cantidad de relleno inorgánico por volumen, el cual varía según la casa comercial. Cerómeros como BelleGlass (Kerr), Targis (Ivoclar Vivadent) reportan mayores cantidades de relleno por volumen que Gradia y Adoro. ${ }^{18}$ Estos valores son similares a los reportados para resinas empacables como la P60. 
En un reporte de investigación de Ivoclar (2004), Gradia (G.C) y Adoro (Ivoclar) presentaron porcentajes de relleno inorgánico por volumen de $35.8 \%$ y $40 \%$, respectivamente, ${ }^{18}$ valores menores comparados con el porcentaje de relleno de las resinas empacables P60 (3M-ESPE) y SureFil (Denstply), que son $62 \%$ y $60 \%{ }^{17}$

Sin embargo, Henao citado por Henostroza (2006) indicó que el cerómero Adoro-Ivoclar presentó un valor de $75 \%$ en volumen y Gradia $64 \% .{ }^{9}$ Esto resultaría en un mejor comportamiento mecánico al compararlo con las resinas empacables.

En este estudio las resinas empacables (P60 y SureFil) fueron divididas en dos grupos, el primero se polimerizó de forma convencional (lámpara LED por 20 segundos cada incremento), el segundo grupo tuvo un curado adicional con calor húmedo (agua en ebullición por 7 minutos) para simular el tratamiento térmico de los cerómeros. Estos últimos fueron manipulados según instrucciones de la casa fabricante y con sus respectivos equipos de polimerización.

El tratamiento térmico mejora el grado de polimerización, así como sus propiedades físicas y mecánicas. ${ }^{6}$ Wendt (1987) sugirió que el tratamiento térmico de las resinas después del fotocurado produce incremento en un $60 \%$ a $70 \%$ de las propiedades mecánicas, especialmente si se les somete a una temperatura entre $120^{\circ} \mathrm{C}$ a $123^{\circ} \mathrm{C}$ durante 5 minutos. ${ }^{19-21}$

Es por esto que los valores de resistencia a la fractura de las resinas empacables con tratamiento térmico adicional, fueron mayores (P60-3M: 273.26Mpa y SureFil-Dentsply: $268.96 \mathrm{Mpa}$ ) comparados con las resinas empacables sin tratamiento térmico (P603M: 254.51Mpa y SureFil- Dentsply: 242.75Mpa)

En este estudio se estandarizó el procedimiento de elaboración de los especímenes de cada material según la norma ISO 4049 y todas las muestras fueron analizadas y observadas por personal externo calificado en el manejo de materiales y pruebas de dureza y resistencia a la fractura ante cargas compresivas

Contrario a la hipótesis formulada en este estudio la cual planteaba que los especimenes en cerómero son más duros y resistentes a la fractura que los especímenes en resina de fotocurado empacable, se concluyó que las resinas empacables con y sin tratamiento térmico poseen un comportamiento físico mecánico superior a los cerómeros estudiados.

El uso de las resinas empacables, P-60 (3M) y SureFil (Dentsply), con técnica de doble curado sometiéndolas en agua a ebullición está indicado para res- tauraciones indirectas, puesto que los resultados de este estudio demostraron superioridad en las propiedades de resistencia a la fractura y dureza. Sin embargo, se sugiere realizar posteriores investigaciones donde se evalúen otras propiedades físicas y mecánicas de estos materiales y técnicas.

Se podría concluir que la resina P-60 (3M) tiene mayor dureza y presenta mayor resistencia a la fractura. Además, esta resina curada con luz más tratamiento térmico es más resistente a la fractura que cuando este material es curado únicamente con luz. Así mismo, la resina P-60 (3M) y SureFil (Dentsply) curadas con luz y con luz y tratamiento térmico son más duras y más resistentes a la fractura que los cerómeros (Adoro- Ivoclar y Gradia-GC)

\section{BIBLIOGRAFÍA}

1. Torres. J. Restauraciones estéticas directas e indirectas a nivel posterior. Bogotá; 2001.

2. Sheibenbogen-Fuchsbrunner A, Manhart J, Kremers L, Kunzelmann KH, Hickel R. Two-year clinical evaluation of direct and indirect composite restorations in posterior teeth. J Prosthet Dent 1999; 82: 391 - 397.

3. Barnes DM, Blank LW, Thompson VP, Holston AM, Gingell JC. A 5 - and 8-year clinical evaluation of a posterior composite resin. Quintessence Int 1991; 22: 143 - 151.

4. Manhart J, Kunzelmann KH, Chen HY, Hickel R. Mechanical properties and wear behaviour of light- cured packable composites resins. Dent Mater 2000; 16: $33-40$.

5. César PF, Miranda WG Jr, Braga RR. Influence of shade and storage time on the flexural strength, flexural modulus, and hardness of composites used for indirect restorations. J Prosthet Dent 2001; 86: 289 - 296.

6. Guzmán. H. Biomateriales odontológicos de uso clínico. 3ra. Edición. ECOE Ediciones: Bogotá; 2003.

7. Davidson-Kaban SS, Davidson CL, Feilzer AJ, de Gee AJ, Erdilek N. The effect of curing light variations on bulk curing and wall-to-wall quality of two types and various shades of resin composites. Dent Mater 1997; 13: 344 352.

8. Loguercio AD, Reis A, Hernandez PA, Macedo RP, Busato AL. Three Year clinical evaluation of posterior packable composite resin restorations. J Oral Rehabil 2006; 33: 144 $-151$.

9. Henostroza G. Estética en Odontología Restauradora. Asociación Latinoamericana de Operatoria Dental y Biomateriales. Madrid: Editorial Médica; 2006.

10. Touati B, Aidan N. Second generation laboratory composite resins for indirect restorations. J Esthet Dent 1997; 9: $108-118$

11. Sabbagh J, Ryelandt L, Bachérius L, Biebuyck JJ, Vreven J, Lambrechts P, Leloup G. Characterization of the inorganic fraction of resin composites. J Oral Rehab 2004; 31: 1090 $-1101$.

12. Da Fonte Porto Carreiro A, Dos Santos Cruz CA, Vergani $\mathrm{CE}$. Hardness and compressive strength of indirect composite resins: effects of immersion in distilled water. $\mathrm{J}$ Oral Rehabil 2004; 31: 1085 - 1089.

13. Dikos MN, McGivney GP, Davis E, Bush PJ, Carter JM. A comparison of the wear resistance and hardness if indi- 
rect composite resins. J Prosthet Dent 2001; 85: 386 - 395.

14. Veranes-Pantoja Y, Autran-Mateu F, Álvarez-Brito R, GilMur FJ. Depth of cure and mechanical properties of experimental light-cured composite resins. RCOE 2005; 10: 151-170.

15. Lovell LG, Lu H, Elliot JE, Stansbury JW. Browman CN. The effect of cure rate on the mechanical properties of dental resins. Dent Mater 2001; 17: 504 - 511.

16. Condon Jr, Ferracane JL. In vitro wear of composite with varied cure, filler level, and filler treatment. J Dent Res 1997; 76: 1405 - 1411.

17. Filtek P60. 3M Dental Products. Manual del Fabricante. 1999.

18. Hopfauf, Simonette. SR Adoro: A modern Indirect Composite. En: Ivoclar Vivadent Report. Lietchtenstein. No 15. Agosto 2004; p. 4 - 48.

19. Leinfelder K. New developments in resin restorative systems. J Am Dent Assoc 1997; 128: 573 - 580.

20. Wendt SL Jr. The effect of heat used on a secondary cure upon the physical properties of three composite resins. I. Diametral tensile strength, compressive strength and marginal dimensional stability. Quintessence Int 1987; 18: 265 - 271

21. Wendt SL Jr. The effect of heat used on a secondary cure upon the physical properties of three composite resins. II. Wear, hardness and color stability. Quintessence Int 1987; 18: $351-356$.

Correo electrónico de los autores:

Antonio Bonilla Nova: dabonillan@gmail.com, Yamile Edilma Franco Rueda: yafraru@gmail.com, Sandra Milena Lara Puerto: samilara@gmail.com, Daniel Armando Peña Acevedo: penaadaniel@hotmail.com, Lucy Margarita Reyes Sarmiento: mare339@hotmail.com, Jhon Jairo Gil Pelaez: jhon_gil@hotmail.com,

jorge Guillermo Díaz Rodríguez: jorgueguillermo12@mail.ustabuca.edu.co
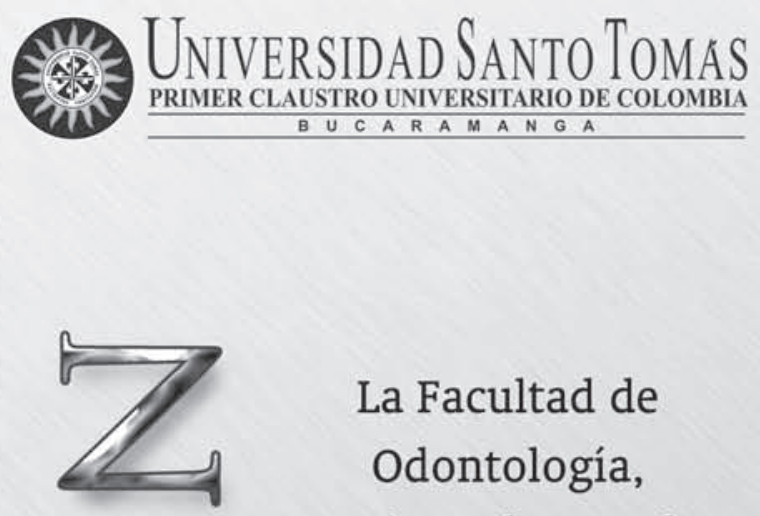

La Facultad de

Odontología, orientada por el pensamiento de

Santo Tomás,

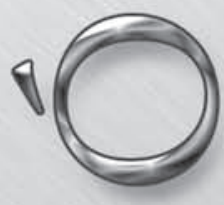
pretende formar

Odontólogos integrales $\mathrm{y} / \mathrm{o}$ especialistas a través

de parámetros

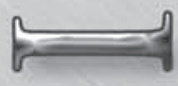
humanísticos, éticos, científicos, biotecnológicos,

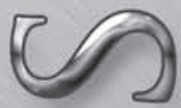
investigativos $\mathrm{y}$ sociales, como recurso humano capaz de intervenir con éxito en el proceso dinámico de la salud y la enfermedad, en el individuo, la familia y la comunidad. 